\title{
METILFENIDATO: UNA REVISIONE
}

\section{ARTICOLO DI REVISIONE}

OLIVEIRA, Vinicius Faustino Lima de ${ }^{1}$, MOREIRA, Danilo José Silva², FONSECA, Juliana Brito da ${ }^{3}$, ROSSI, Karoline ${ }^{4}$, VASCONCELOS, Suzana dos Santos ${ }^{5}$, DIAS, Claudio Alberto Gellis de Mattos ${ }^{6}$, OLIVEIRA, Euzébio de ${ }^{7}$, DENDASCK, Carla Viana ${ }^{8}$, ARAÚJO, Maria Helena Mendonça de ${ }^{9}$, FECURY, Amanda Alves ${ }^{10}$

OLIVEIRA, Vinicius Faustino Lima de. Et al. Metilfenidato: una revisione. Revista Científica Multidisciplinar Núcleo do Conhecimento. An 06, Ed. 05, Vol. 01, pp. 05-14. Mai 2021. ISSN: 2448-0959, Lien d'accès: https://www.nucleodoconhecimento.com.br/sante/metilfenidato-una-revisione, DOI: 10.32749/nucleodoconhecimento.com.br/sante/metilfenidato-una-revisione

\section{RÉSUMÉ}

Le méthylphénidate (MFD) est un médicament dérivé des amphétamines qui stimule le système nerveux central (SNC), favorisant une attention accrue, moins de distraction et un sens accru de la motivation. Cette étude vise à décrire les principales caractéristiques pharmaceutiques de la MFD, à mettre en évidence les indications

\footnotetext{
${ }^{1}$ Médecin spécialiste. I'Université fédérale d'Amapá (UNIFAP).

${ }^{2}$ Médecin spécialiste. I'Université fédérale d'Amapá (UNIFAP).

${ }^{3}$ Universitaire en médecine. I'Université fédérale d'Amapá (UNIFAP).

${ }^{4}$ Universitaire en médecine. l'Université fédérale d'Amapá (UNIFAP).

${ }^{5}$ Universitaire en médecine. I'Université fédérale d'Amapá (UNIFAP).

${ }^{6}$ Théologien, docteur en psychanalyse clinique. II travaille depuis 15 ans avec la Méthodologie Scientifique (Méthode de Recherche) dans I'Orientation de Production Scientifique pour les étudiants en Master et Doctorat. Spécialiste en études de marché et recherche en santé, doctorant en communication et sémiotique (PUC SP).

${ }^{7}$ Biologiste, Ph.D. en maladies tropicales, professeur et chercheur du cours d'éducation physique, Université fédérale du Pará (UFPA).

${ }^{8}$ Théologien, Ph.D. en psychanalyse clinique. II travaille depuis 15 ans avec la méthodologie scientifique (méthode de recherche) dans le cadre de l'orientation de production scientifique des étudiants à la maîtrise et au doctorat. Spécialiste de l'étude de marché et de la recherche axée sur la santé.

${ }^{9}$ Docteur, Master en enseignement et sciences de la santé, professeur et chercheur du cours de médecine du campus de Macapá, Université fédérale d'Amapá (UNIFAP).

${ }^{10}$ Biomédical, PhD en maladies tropicales, professeur et chercheur du cours médical du campus de Macapá, Université fédérale d'Amapá (UNIFAP), Pro-Recteur de la recherche et des études supérieures (PROPESPG) de I'Université fédérale d'Amapá (UNIFAP).

RC: 84390
}

Link di accesso: https://www.nucleodoconhecimento.com.br/salute/metilfenidato-una- 
cliniques pour l'utilisation de la MFD et à détailler les effets indésirables possibles découlant de la consommation de MFD. Pour cela, nous avons mené une recherche dans les bases de données ScienceResearch.com, SciELO, LILACS, PubMed et EMBASE et dans les référentiels institutionnels de l'Université fédérale de São Paulo, de l'Université fédérale du Minas Gerais et de l'Université fédérale de Ceará, en utilisant les mots clés et les associations " méthylphénidate ", "ritalin », " effets » et " effects ». Il a été démontré que le médicament se compose d'un mélange racemic formé par quatre stereoisomers, et le composé pharmacologiquement actif est racemate dextrogyl (d,I)-treo-MFD. Il agit sur le blocus des transporteurs de dopamine et de noradrénaline du SNC, favorisant les effets qui justifient sa prescription pour traiter le trouble déficitaire de l'attention avec hyperactivité (TDAH). Ces dernières années, sa consommation à des fins non thérapeutiques a augmenté. Certains effets indésirables de son utilisation ont été rapportés, et l'insomnie, l'irritabilité et le mal de tête ont été rapportés.

Mots-clés: Méthylphénidate, Dopamine, Noradrénaline.

\section{INTRODUCTION}

Le méthylphénidate (MFD) est un stimulateur du système nerveux central (SNC) de la pipéidine, un type d'amphétamine (PAPA, 2013). La MFD a une consommation plus élevée que la somme de tous les autres psychostimulants, étant les plus consommés dans le monde (SALVIANO, 2015).

Au début des années 1990, à l'échelle mondiale, 2,8 tonnes de MFD ont été produites. En 2000, cette valeur a été portée à 16 tonnes et, dix ans plus tard, la marque statistique a atteint 43 tonnes. Au Brésil en 2011, $413 \mathrm{~kg}$ de MFD (CALIMAN; DOMITROVIC, 2013).

II est prescrit pour les personnes diagnostiquées avec le trouble déficitaire de l'attention avec hyperactivité (TDAH) et pour la narcolepsie (CALDEIRA, 2015). Au Brésil, MFD est disponible pour être commercialisé sous les noms Ritalina ${ }^{\circledR}$, Ritalina $L A \circledast$ et Concerta $\AA$, tous avec prise orale. La dynamique de libération de mfd dans le RC: 84390 Link di accesso: https://www.nucleodoconhecimento.com.br/salute/metilfenidato-una- 
corps ont des options immédiates et lentes d'action, telles que Ritalin $®$ qui a l'effet immédiat, alors que Ritalin $L A \circledast$ et Concerta ${ }^{\circledR}$ présentent des formules à action longue (BATISTA, 2015).

Au début du traitement par MFD, les doses indiquées aux patients en bas âge vont de 5 à $20 \mathrm{mg} / \mathrm{jour}$, avec des ajustements possibles, et des incréments peuvent être faits jusqu'à la dose quotidienne maximale de $60 \mathrm{mg} / \mathrm{jour}$. Une autre option pour déterminer la dose quotidienne commence par deux apports quotidiens de 0,3 mg/kg et, selon les besoins du cas, une augmentation de $0,1 \mathrm{mg} / \mathrm{kg} /$ dose peut être faite chaque semaine et ne doit pas dépasser $2 \mathrm{mg} / \mathrm{kg} / \mathrm{jour}$. En raison du manque d'études destinées aux enfants de moins de 6 ans, l'utilisation de la MFD dans cette population n'est pas indiquée. Chez les adultes, la dose quotidienne de MFD varie de 10 à 72 mg (FARIA, 2017).

Le MFD offre une attention accrue, ce qui se traduit par moins de distraction, et un sens accru de la motivation, conduisant à l'intérêt pour la réalisation des objectifs et des actions (CÂNDIDO, 2018). Au Brésil, l'utilisation légale de la MFD n'est que pour traiter les maladies et les troubles. Ce médicament fait partie de la Liste A3 des psychotropes dépendant d'un contrôle spécial, et son acquisition est conditionnée à la recette jaune spéciale et au maintien de la prescription en pharmacie au moment de la vente (FARIA, 2017).

\section{OBJECTIFS}

Décrire les principales caractéristiques pharmaceutiques de la MFD. Mettre en évidence les indications cliniques pour l'utilisation de MFD. Détailler les effets indésirables possibles découlant de la consommation de MFD.

\section{MÉTHODE}

Les références utilisées ont été obtenues par la recherche dans les bases de données : ScienceResearch.com, SciELO, LILACS, PubMed, EMBASE, Dépôt institutionnel de l'Université fédérale de São Paulo, Dépôt institutionnel de l'Université fédérale du RC: 84390 Link di accesso: https://www.nucleodoconhecimento.com.br/salute/metilfenidato-una- 
Minas Gerais et Dépôt institutionnel de l'Université fédérale de Ceará, en utilisant les mots clés et associations suivants : « méthylphénidate », « ritalin », « effets » et « effects ».

La méthode de sélection des documents était la suivante : identification, dépistage, admissibilité et inclusion. Pour être incluses dans cet examen, les études devaient avoir un texte complet accessible dans les bases de données étudiées, avec un contenu pertinent concernant le MFD, ainsi que d'être disponibles dans l'une des langues suivantes: portugais, anglais ou espagnol. Exclusivement dans la plate-forme PubMed, un filtre temporel a été utilisé dans lequel les publications des 5 dernières années ont été utilisées, en plus d'utiliser des œuvres liées à l'homme.

L'identification a été la fin de la réunion d'études existantes dans la littérature sur la MFD dans les bases de données. La présélection a été effectuée après une analyse rapide du titre et de l'résumé des publications, portant ces études à la catégorie des personnes admissibles. En fin de compte, toutes les œuvres admissibles ont été entièrement lues et, par conséquent, exclues de celles qui n'étaient pas alignées sur les objectifs du cet examen et qui n'ont peut-être pas été exclues aux étapes précédentes. Enfin, les étapes approuvées ont été incluses dans toutes les étapes précédentes de la méthode appliquée.

\section{RÉSULTATS}

La recherche sur le MFD dans les bases de données a donné lieu à 1038 articles au stade méthodologique de l'identification. Lors du dépistage, 981 ont été exclus, puisque seulement $5,49 \%$ des études étaient admissibles. Enfin, avec la lecture complète de tous les documents admissibles, 37 documents ont été inclus et 35,09\% des documents admissibles ont été jetés.

\section{PHARMACOLOGIE MFD}

Le MFD, dont la nomenclature IUPAC (International Union of Pure and Applied Chemistry) est l'acétate Metil 2-fenil-2(piperidil), a deux carbones chiraux et la formule RC: 84390

Link di accesso: https://www.nucleodoconhecimento.com.br/salute/metilfenidato-una- 
chimique $\mathrm{C}_{14} \mathrm{H}_{19} \mathrm{NO}_{2}$. II se compose d'un mélange racemic formé par quatre stereoisomers, qui sont $d$-treo-MFD, l-treo-MFD, $d$-erythro-MFD et l-eritro-MFD. Le composé pharmacologiquement actif est le dextrogyl racemate (d,I)-treo-MFD, qui réagit avec des récepteurs de dopamine dans le corps strié et fournit la stimulation noradrenergic dans le cortex (BATISTA, 2015; FREESE et al., 2012; LINHARES, 2012).

MFD est un agoniste indirect de dopamine et de noradrénaline, influençant intensément l'action de ces neurotransmetteurs dans les centres moteurs et l'attention (BATISTA, 2015 ; BATISTELA, 2011). II agit en inhibant la recapture de la dopamine par les neurones présinaptiques des régions corticales et subcortiques (FREESE et al., 2012).

\section{APPLICATIONS THÉRAPEUTIQUES DU MFD}

MFD est le psychostimulant de première ligne pour l'approche pharmacologique du TDAH et pour le traitement de la narcolepsie, un trouble du sommeil très rare. II est également utilisé à des fins récréatives, esthétiques et cognitives améliorées liées à l'augmentation de la productivité scolaire et du travail (BARROS; ORTEGA, 2011; BATISTELA, 2011; BRANT; CARVALHO, 2012; CALDEIRA, 2015; CÂNDIDO, 2018; SALVIANO, 2015).

II est rapporté que l'association de MFD avec l'inhibiteur sélectif de recapture de sérotonine (ISRS) était efficace dans la thérapie des symptômes du syndrome de Gilles de la Tourette (PASSOS ; LÓPEZ, 2010), ainsi que pour le traitement du déficit cognitif chez un patient victime d'une lésion cérébrale traumatique (TCE) (ROCHA; MALLOY-DINIZ; HARA, 2006).

MFD est également utilisé chez les patients adultes atteints de néoplasmes avancés, afin de traiter la somnolence due à l'utilisation d'opioïdes, symptômes dépressifs, delirium hypoactif et la fatigue due au cancer. II y a une description que MFD est une alternative thérapeutique pour des patients diagnostiqués avec la manie aiguë (CAMPOS et autres, 2016; LÓPEZ-GARCÍA; HEGERL, 2013; PAPA, 2013). 


\section{EFFETS INDÉSIRABLES DE L'UTILISATION DU MFD}

Les enfants et les adolescents ont comme principaux effets indésirables insomnie et diminution de l'appétit, étant directement proportionnelle aux doses de MFD ingéré, en plus de présenter la xérostomie et l'irritabilité. La participation grave de l'état psychiatrique peut se produire, potentialant ou causant des désordres psychotiques ou des épisodes de manie et de tics. Des effets tels que des douleurs abdominales et des maux de tête sont importants, ainsi qu'une légère augmentation de la fréquence cardiaque (BATISTA, 2015; FARIA 2017; LOUZÃ; MATTOS, 2007; PASTURA; MATTOS, 2004).

Avec l'utilisation prolongée, les effets secondaires tels que la réduction de la taille et le développement de la dépendance chimique dans le patient (BRANT; CARVALHO, 2012; PASTURA; MATTOS, 2004). En outre, on observe qu'il peut y avoir des augmentations supérieures à $10 \mathrm{mmHg}$ dans la pression artérielle des utilisateurs de MFD (CÂNDIDO, 2018).

Une étude utilisant des cobayes animaux indique que l'administration aiguë et chronique de MFD nuit à l'acide désoxyribonucléique (ADN) dans le corps strié et hippocampe (SALVIANO, 2015). II y a des rapports que MFD cause également la nausée, la diminution de vomissement de la croissance et du taux de poids, des arythmies, des toux et des hallucinations, qui sont visuelles et tactiles dans la nature liée aux insectes, aux reptilils ou aux vermines (BATISTA, 2015). Chez les enfants et les jeunes adultes ayant reçu un diagnostic de TDAH qui utilisent le MFD, la psychose survient chez 1 personne sur 660 (MORAN et al., 2019).

L'alopécie est signalée pour l'utilisation de MFD (NÚÑES-GARCES; SÁNCHEZGAYANGO; ROMERO-PÉREZ, 2018), et ce MFD à libération lente peuvent être liés au développement du phénomène Raynaud, une implication caractérisée par une vasoconstriction excessive des artérioles qui irriguent la peau (OTERO et al., 2012).

Grazina et al. (2018) décrivent la fracture fémorale chez une jeune fille diagnostiquée avec le TDAH traité avec le MFD, associant le médicament comme cause possible. RC: 84390 Link di accesso: https://www.nucleodoconhecimento.com.br/salute/metilfenidato-unarecensione 
Dans cette étude, ils soulignent que la densité osseuse d'un enfant utilisant la MFD est différente de celle qui n'utilise pas, ce qui suggère que le médicament augmente le risque de fracture.

Chez les rats, qui ont un tissu pulmonaire histologiquement compatible avec l'homme, l'administration de MFD a causé des dommages au parenchyme pulmonaire par la destruction de septa alvéolaire (RAPELLO et al., 2015). Le MFD peut être considéré comme un agent induisant des différenciations morphologiques chez les glandes salivaires des rats (LIMA et al., 2016).

L'association de MFD avec le développement de la sclérose systémique est décrite dans les patients présentant la maladie autoimmune et vasopathique suspectée (MERIDOR; LEVY, 2019). II y a des rapports du cas d'un patient de quatre décennies de vie qui a subi la chirurgie bariatrique avec le pontage gastrique laparoscopic rouxlike qui avait utilisé le MFD pendant environ un an, et après deux semaines de chirurgie ont commencé à présenter des symptômes de toxicité de MFD, tels que la paranoïa et les hallucinations auditives (LUDVIGSSON ; HAENNI, 2016).

\section{DISCUSSION}

\section{MÉCANISME DE FONCTIONNEMENT MFD ET SA MÉTABOLISATION}

Bien que les caractéristiques pharmacologiques du MFD soient bien établies, son mécanisme de fonctionnement n'est pas encore entièrement compris (BRUNELLI, 2013; LINHARES, 2012).

On sait que le MFD agit sur les voies dopaminergiques et noradrenergiques des régions corticales et subcortical du cerveau, principalement dans le corps strié, se liant aux transporteurs de dopamine et de noradrénaline, les bloquant. II en résulte une plus grande disponibilité dans les fissures synaptiques locales, ce qui génère des effets excitatifs qui reflètent les avantages promus par le MFD (BATISTELA, 2011; BRUNELLI, 2013; CÂNDIDO, 2018; FERNÁNDEZ, 2018; PALHARES, 2015; SALVIANO, 2015). 
Les principaux transporteurs de dopamine établis sont DAT1 et DRD4. II y a un rapport dans la littérature que quelques mutations dans les gènes codant ces transporteurs sont associées à la réponse accrue au MFD, une réponse qui est également liée aux facteurs sociaux et cliniques (BATISTA, 2015).

L'augmentation du niveau de dopamine due à la drogue se produit dans les zones du SNC, qui sont sensibles à l'action des drogues d'abus (FARIA, 2017).

La noradrénaline a NAT comme transporteur, qui est le mécanisme primaire de l'inactivation dopaminergique de voie. Lorsqu'il est bloqué, il augmente les niveaux pré-sinaptitic de noepinephrine, ayant pour résultat une plus grande activation de la voie de noradrenegy (LINHARES, 2012).

La dopamine agit directement dans la modulation des fonctions cognitives et motrices, la consolidation de la mémoire, la promotion de l'attention, en plus du traitement de certaines informations environnementales d'une importance primordiale pour la survie, l'exécution de ces fonctions, en partie, sur les récepteurs D1 (fonction excitatrice) et D2 (fonction inhibante) du cortex préfrontal. Par conséquent, on suppose que le déficit dans sa fonction cause l'aspect de l'inattention et de l'hyperactivité, deux signes cliniques compatibles avec l'image du TDAH (BATISTELA, 2011; LIANG et al., 2018; LINHARES, 2012; OCHI; VIEIRA, 2016).

En ce qui concerne la Noradrénaline, on sait qu'elle agit dans des zones qui influencent les stimuli d'inhibition comportementale. Ainsi, on en déduit que le déficit de sa fonction peut conduire à l'incapacité de contrôler les impulsions, une autre caractéristique du TDAH (LIANG et coll., 2018; LINHARES, 2012; OCHI; VIEIRA, 2016).

Il existe trois formulations MFD disponibles sur le marché: Ritalin $\Theta$, Ritalin $L A \circledast$ et Concerta $L A \circledast$, qui varient par rapport à la demi-vie, le temps de libération et la durée d'action. Le premier consiste en une formulation d'action rapide, favorisant les pics MFD environ 1 à 3 heures après l'ingestion, avec une demi-vie entre 2 à 3 heures et la durée d'action entre 3 et 6 heures. Ces deux derniers consistent en des formulations d'action rapide, qui favorisent les pics de MFD environ 3 à 4 heures après l'ingestion, RC: 84390 Link di accesso: https://www.nucleodoconhecimento.com.br/salute/metilfenidato-unarecensione 
avec une demi-vie de 4 heures et une durée d'action allant de 8 à 12 heures. Ce qui va différer d'eux est la quantité de doses qui peuvent être prises par jour: Ritalin LA $®$ peut être pris 1 ou 2 fois par jour; La Concerta $\AA^{\circ} 1$ fois par jour (LINHARES, 2012; LOUZÃ; MATTOS, 2007; PAPA, 2013).

L'absorption de MFD se produit complètement, et peut facilement traverser la barrière hématocéphale due à sa liposolubility (LINHARES, 2012).

Environ 48 à 96 heures, MPH est métabolisé par le système microsomique hépatique, par un processus d'hydrolyse qui génère de l'acide ritalin, substrat éliminé du corps par excrétion urinaire. Le reste du médicament qui n'est pas métabolisé sera éliminé avec la sécrétion de bile (LINHARES, 2012; PAPA, 2013).

La métabolisation du MFD dans le foie se produit par l'action des carboxylésases (CES) - principalement CES1. Les mutations dans le gène codant l'enzyme sont liées à un déficit dans le métabolisme du médicament, susceptible l'utilisateur au développement des réactions défavorables (BATISTA, 2015).

\section{SÉCURITÉ D'UTILISATION}

Parce qu'il s'agit d'un dérivé de l'amphétamine, MFD provoque plusieurs discussions dans la littérature sur la capacité de ce médicament à causer une dépendance chimique et une utilisation excessive chez les utilisateurs, ce qui est encore controversé. La rareté des cas de dépendance et d'abus chimiques suggère un faible risque de la part du méthylphénidate (BRANT; CARVALHO, 2012; FERNÁNDEZ, 2018; PALHARES, 2015; PASTURA; MATTOS, 2004).

II est recommandé d'évaluer l'existence possible d'instabilité émotionnelle chez le patient avant de prescrire la MFD, ce qui est dû au risque que ces personnes ont d'augmenter spontanément les doses du médicament (BRANT; OAK, 2012).

Les personnes qui ont déjà eu des comorbidités telles que l'hypertension artérielle systémique, les arythmies cardiaques, la dépression, l'épilepsie et le trouble bipolaire 
devraient être traitées avec prudence concernant l'utilisation de MFD. Dans les deux premiers cas, les patients devraient être surveillés au fil du temps pour détecter les complications possibles. Dans le troisième cas, mfd est possible avec des antidépresseurs. Dans les deux derniers cas, il est rapporté qu'il y a sûreté concernant l'utilisation de MFD, à condition que le patient ait son état clinique stabilisé et correctement traité (LOUZ̃̃ ; MATTOS, 2007).

Parmi les formulations disponibles du médicament, la formulation de libération prolongée (Concerta $\left.{ }^{\circledR}\right)$, en plus d'être la plus pratique, car elle a une durée plus longue, est également la plus sûre, car elle empêche l'apparition d'effets nocifs découlant de l'augmentation soudaine des niveaux de méthylphénidate dans le corps, sans perdre son efficacité (FARIA, 2017; LOUZÃ; MATTOS, 2007; PAPA, 2013).

Bien que le MFD soit inclus dans la législation brésilienne dans la liste A3 des substances psychotropes, le méthylphénidate n'a pas la même pharmacotoxicité que les autres substances de ce groupe (méthamphétamine, phéncyclidine, dronabinol, entre autres), ce qui lui donne une plus grande sécurité d'utilisation (CARLINI et al., 2003).

\section{CONSOMMATION DE MFD}

Depuis la troisième décennie du XXe siècle, la classe des médicaments dérivés des amphétamines a suscité l'intérêt des industries pharmaceutiques pour le traitement des troubles du comportement, compte tenu de sa capacité à stimuler le SNC (CÂNDIDO, 2018; FERNÁNDEZ, 2018).

Le MFD a été synthétisé en 1944 par Leandro Panizzon et en 1954 a été breveté sous le nom de Ritalin (CÂNDIDO et coll., 2019). Même avec son existence, jusque-là, on ne savait pas que son utilisation pouvait apporter des améliorations à l'image du TDAH. Cela n'a été établi que dans les années 1960 et 1970 lorsque des études ont prouvé des avantages pour les enfants atteints de troubles du comportement (BRANT; CARVALHO, 2012; GONÇALVES; PEDRO, 2018). 
Malgré cela, la consommation de mfd était déjà établie à grande échelle dans le monde entier, étant utilisée pour traiter la fatigue chronique et la narcolepsie, en plus d'être utilisée pour la perte de poids, l'amélioration des performances athlétiques et la performance intellectuelle (BRANT; CARVALHO, 2012; BRUNELLI, 2013). Selon Barros et Ortega (2011), les utilisations non thérapeutiques sont appelées récréatives, lorsqu'elles sont utilisées pour améliorer le temps d'éveil et la volonté de pratiquer les loisirs, les activités esthétiques, lorsqu'elles sont utilisées pour la perte de poids, et l'amélioration cognitive.

Dans les années 1960, en vérifiant l'amélioration de l'image clinique du TDAH avec l'administration du MFD, la communauté scientifique est arrivée à un consensus sur l'utilisation du médicament pour diagnostiquer ce trouble, qui a été initialement établi pour les enfants, mais qui, au fur et à mesure que d'autres études ont été développées, est devenu efficace aussi pour les adolescents et les adultes. Si l'individu avec le TDAH suspecté a présenté l'amélioration après l'utilisation du MFD, on a supposé qu'il a eu la maladie (BRUNELLI, 2013; GONÇALVES; PEDRO, 2018). Cet aspect, ainsi que l'élargissement des critères de diagnostic du TDAH et l'augmentation conséquente de l'incidence du TDAH, ont contribué à l'exacerbation de sa consommation ces dernières années (GONÇALVES; PEDRO, 2018; SALVIANO, 2015).

Toutefois, ce qui attire le plus l'attention dans le scénario actuel, c'est l'appropriation de la consommation de MFD à des fins non thérapeutiques, principalement par les personnes de plus de 19 ans, Étudiants du cours, les étudiants, les cadres supérieurs, les entrepreneurs et les professionnels de la santé comme principaux utilisateurs, étant la principale motivation à rechercher des performances plus élevées au cours de leurs activités (BRANT; CARVALHO, 2012; CALIMAN; RODRIGUES, 2014; CÂNDIDO et al., 2019; GONÇALVES; PEDRO, 2018; MORGAN et al., 2017; SALVIANO, 2015).

Il convient de souligner l'augmentation de la consommation chez les adolescents, pour une utilisation liée à la diminution de l'appétit et de la fatigue, en plus du 
neuroenhancement (CÂNDIDO et al., 2019; GONÇALVES; PEDRO, 2018; SALVIANO, 2015). L'étude menée par Cândido et al. (2019) a révélé que sur les étudiants évalués qui utilisaient le méthylphénidate pour le neuroenhancement, environ un tiers d'entre eux ont acquis le médicament sans ordonnance. Les mêmes auteurs appellent l'attention sur la nécessité d'intervenir sur cette pratique, car il n'y a pas de preuve de l'efficacité de la MFD à cette fin.

\section{DOSAGE DE MFD ET SA RELATION AVEC LES EFFETS INDÉSIRABLES DU MÉDICAMENT}

Certaines études montrent que le développement d'effets indésirables dus à l'utilisation de MFD, tels que l'insomnie et la diminution de l'appétit, est conditionné par la posologie administrée, et ces événements peuvent revenir au fil du temps ou avec une dose diminuée (BATISTA, 2015; BATISTELA, 2011; LOUZÃ; MATTOS, 2007; PASTURA; MATTOS, 2004).

Holmskov et al. (2017), sur la base d'une étude sur les effets indésirables gastrointestinaux pendant le traitement par méthylphénidate, n'a trouvé aucune différence dans le risque d'effets indésirables lorsqu'on considère la dose, le type ou la durée de l'administration.

On contacte une faible fréquence de développement d'effets indésirables chez les utilisateurs du médicament et, dans la plupart des cas, ils sont légers ou modérés (SALVIANO, 2015).

Dans le cas des enfants, le traitement du TDAH se fait avec des doses de 5 à 20 $\mathrm{mg} / \mathrm{jour}$, avec des ajustements au besoin (FARIA, 2017).

$\mathrm{Papa}^{1}$ souligne qu'aucun changement cardiovasculaire ne se trouve avec des doses inférieures à $30 \mathrm{mg}$. Ceci est important pour la thérapie de la maladie, puisqu'il y a des rapports des changements cardio-vasculaires avec l'utilisation de MFD (FARIA, 2017; HENNISSEN et al., 2017; SALVIANO, 2015). 
Pour atténuer les malaises générés par certains effets indésirables, il y a des recommandations à suivre : cesser l'utilisation de la MFD avec l'émergence de tout événement indésirable, afin de vérifier si le médicament était l'agent causal. En cas d'aggravation coïncident avec les niveaux de pointe de MFD dans le corps, il peut être proposé l'utilisation de la formulation à longue libération. Dans l'insomnie, éviter l'utilisation de MFD près de l'heure du coucher, augmenter la posologie le matin et diminuer la nuit, sont des mesures qui sont efficaces. Pour la perte d'appétit, le patient peut opter pour la formulation à action courte, afin de gérer le temps d'action du médicament et, par conséquent, l'effet secondaire promu par celui-ci (PASTURA; MATTOS, 2004).

\section{CONCLUSION}

Les effets de la MFD proviennent de l'inhibition des récepteurs neurotransmetteurs tels que la dopamine et la noradrénaline dans les régions corticales et subcortical liées au contrôle moteur et à l'attention. Avec cette inhibition, les neurotransmetteurs sont en plus grande quantité de la fente synaptique, causant les effets du médicament.

MFD peut générer la dépendance chimique, car il est dérivé des amphétamines, mais dans la littérature il y a peu de cas de dépendance résultant de son utilisation.

MFD est indiqué pour les patients diagnostiqués avec le TDAH, mais peut également être utilisé à d'autres fins, y compris le traitement de la narcolepsie, l'amélioration cognitive, les loisirs, l'esthétique, une approche alternative à la manie aiguë, et le traitement des symptômes des patients atteints de néoplasmes avancés.

L'utilisation de MFD peut causer des effets secondaires, dont les principaux sont l'insomnie et le manque d'appétit, et ces effets sont directement proportionnels aux doses ingérées.

La littérature montre que le MFD est efficace dans le traitement du TDAH, mais ne indique pas clairement s'il cause la dépendance, ni n'est influencé négativement sur la croissance et le gain de poids des enfants. 


\section{RÉFÉRENCES}

BARROS, D.; ORTEGA, F. Metilfenidato e Aprimoramento Cognitivo Farmacológico: representações sociais de universitários. Saúde Soc, v. 20, n. 2, p. 350-63, 2011.

BATISTA, J. M. N. Estudo farmacoterapêutico e farmacogenético em crianças e adolescentes com transtorno do déficit de atenção e hiperatividade tratados com metilfenidato. Tese de Doutorado - Universidade de Federal do Ceará. Fortaleza, p. 95. 2015.

BATISTELA, S. Efeitos da administração aguda de diferentes doses do metilfenidato sobre a cognição de jovens saudáveis. Tese (Mestre em Ciências) - Universidade Federal de São Paulo. São Paulo, p. 88. 2011.

BRANT, L. C.; CARVALHO, T. R. F. Metilfenidato: medicamento gadget da contemporaneidade. Interface comun saúde educ, v. 16, n. 42, p. 623-36, 2012.

BRUNELLI, L. F. O Metilfenidato (RITALINA®) na escola: Percepção dos educadores da rede de Ensino fundamental I e II. Dissertação (Mestrado em Ciências Humanas e Sociais Aplicadas) Universidade Federal de Minas Gerais. Belo Horizonte, p. 100. 2013.

CALDEIRA, C. M. P. Frequência do transtorno de déficit de atenção e hiperatividade (TDAH) e os problemas psicológicos associados em crianças e adolescentes. Monografia (Especialista em Neurociências) - Universidade de Federal do Ceará. Fortaleza. p. 64. 2015.

CALIMAN, L. V.; DOMITROVIC, N. Uma análise da dispensa pública do metilfenidato no Brasil: o caso do Espírito Santo. Physis, v. 23, n. 3, p. 879-902, 2013.

CALIMAN, L. V.; RODRIGUES, P. H. P. A experiência do uso de metilfenidato em adultos diagnosticados com TDAH. Psicol estud, v. 19, n. 1, p. 125-134, 2014. 
CAMPOS, M. P. O. et al. Fadiga relacionada ao câncer: uma revisão. Rev Assoc Med Bras, v. 57, n. 2, p. 211-219, 2016.

CÂNDIDO, R. C. F. Metilfenidato de liberação imediata para o transtorno do déficit de atenção e hiperatividade (TDAH) em adultos - revisão sistemática. Dissertação (Mestrado em Medicamentos e Assistência Farmacêutica) - Universidade Federal de Minas Gerais. Belo Horizonte, p. 147. 2018.

CÂNDIDO, R. C. F. et al. Perini E, Pádua CM, Junqueira DR. Prevalência e fatores associados ao uso de metilfenidato para neuroaprimoramento farmacológico entre estudantes universitários. Psicol conoc Soc, v. 18, p. 1-7, 2019.

CARLINI, E. A. et al. Metilfenidato: influência da notificação de receita A (cor amarela) sobre a prática de prescrição por médicos brasileiros. Rev Psiquiatr Clín, v. 30, n. 1, p. 11-20, 2003.

FARIA, J. C. M. Desempenho acadêmico de estudantes com diagnóstico de transtorno do déficit de atenção e hiperatividade (TDAH) em uso de metilfenidato - revisão sistemática. Dissertação (Mestrado em Medicamentos e Assistência Farmacêutica) - Universidade Federal de Minas Gerais. Belo Horizonte, p. 77.2017.

FERNÁNDEZ, L. C. S. Psicoestimulantes para el TDAH: análisis integral para una medicina basada en la prudencia. Rev Asoc Esp Neuropsiq, v. 38, n. 133, p. 301330. 2018.

FREESE, L. et al. Uso não médico de metilfenidato: uma revisão. Trends psychiatry psychother, v 34, n. 2, p. 110-115, 2012.

GONÇALVES, C. S.; PEDRO, R. M. L. R. "Drogas da Inteligência?": Cartografando as controvérsias do consumo da Ritalina para o aprimoramento cognitivo. Psicol conoc Soc, v. 8, n. 2, p 71-94, 2018. 
GRAZINA, R. et al. Fractura por estrés del cuello del fémur en unaniña hiperactiva tratada con metilfenidato - Reporte de caso. Rev Asoc Argent Ortop Traumatol, v. 83 , n. 1 , p. 45-49, mar. 2018.

HENNISSEN, L. et al. Cardiovascular Effects of Stimulant and Non-Stimulant Medication for Children and Adolescents with ADHD: A Systematic Review and MetaAnalysis of Trials of Methylphenidate, Amphetamines and Atomoxetine. CNS drugs, v. 31, n. 3, p. 199-215, 2017.

HOLMSKOV, M. et al. Gastrointestinal adverse events during methylphenidate treatment of children and adolescents with attention déficit hyperactivity disorder: $A$ systematic review with meta-analysis and Trial Sequential Analysis of randomised clinical trials. PLos ONE, v. 12, n. 6, p. 1-18, 2017.

LIANG, E. F. et al. The Effect of Methylphenidate and Atomoxetine on Heart Rate and Systolic Blood Pressure in Young People and Adults with Attention-Deficit Hyperactivity Disorder (ADHD): Systematic Review, Meta-Analysis, and MetaRegression. Int J Environ Res Health Public, v. 17, n. 1789, p 1-16, 2018.

LIMA, K. S. et al. Efeitos do metilfenidato sobre as glândulas salivares maternas de camundongos. Rev Odontol UNESP, v. 45, n. 6, p. 316-321, 2016.

LINHARES, M. I. Estudo da Ritalina ${ }^{\circledR}$ (cloridrato de metilfenidato) sobre 0 sistema nervoso central de animais jovens e adultos: aspectos comportamentais e neuroquímicos. Dissertação (Mestrado em Farmacologia) Universidade Federal do Ceará. Fortaleza, p. 145. 2012.

LÓPEZ-GARCÍA, P.; HEGERL, U. Tratamiento de la manía aguda com metilfenidato: propuesta terapêutica basada en un nuevo modelo fisiopatológico. Rev psiquiatr salud ment, v. 6, n. 2, p. 93-94, 2013.

LOUZÃ, M. R.; MATTOS, P. Questões atuais no tratamento farmacológico do TDAH em adultos com metilfenidato. J bras psiquiatr, v. 56, n. 1, p. 53-56, 2007. 
LUDVIGSSON, M.; HAENNI, A. Methylphenidate toxicity after Roux-en-Y gastric bypass. Surg Obes Relat Dis, v. 12, n. 5, p. 55-57, 2016.

MERIDOR, K.; LEVY, Y. Systemic sclerosis induced by CNS stimulants for ADHD: A case series and review of the literature. Autoimmun Rev, v. 19, n. 1, p. 1-3, 2019.

MORAN, L. V. et al. Psychosis with Methylphenidate or Amphetamine in Patients with ADHD. N Engl J Med, v. 380, n. 12, p. 1128-1138, 2019.

MORGAN, H. L. et al. Consumo de Estimulantes Cerebrais por Estudantes de Medicina de uma Universidade do Extremo Sul do Brasil: Prevalência, Motivação e Efeitos Percebidos. Rev bras educ med, v. 41, n. 1, p. 102-109, 2017.

NÚÑES-GARCES, M.; SÁNCHEZ-GAYANGO, A.; ROMERO-PÉREZ, C. Alopecia reversible secundaria a metilfenidato OROS. Rev colomb psiquiatr. 2018. DOI: 10.1016/j.rcp.2018.09.003.

OCHI, N. O.; VIEIRA, G. L. Efeitos do metilfenidato no desempenho motor de crianças com TDAH. Revista Saúde e Pesquisa, v. 9, n. 1, p. 93-96, 2016.

OTERO, M. I. et al. Metilfenidato y fenómeno de Raynaud secundario. Semergen, v. 39, n. 6, p. 330-334, 2012.

PALHARES, J. P. P. "Eu tomo medicamentos para estudar": compreendendo a experiência com metilfenidato entre estudantes universitários. Dissertação (Mestrado em Medicamentos e Assistência Farmacêutica - Universidade de Federal de Minas Gerais. Belo Horizonte, p. 86. 2015.

PAPA, M. P. Uso del metilfenidato en pacientes con cáncer avanzado. Rev Med Urug, v. 29 , n. 1, p. 58-63, 2013.

PASSOS, R. B. F.; LÓPEZ, J. R. R. A. Síndrome de Gilles de la Tourette associada ao transtorno de déficit de atenção com hiperatividade: resposta clínica satisfatória a 
inibidor seletivo da recaptura de serotonina e metilfenidato. J bras psiquiatr, v. $59, \mathrm{n}$. 2, p. 160-162, 2010.

PASTURA, G; MATTOS, P. Efeitos colaterais do Metilfenidato. Revista de Psiquiatria Clínica, v. 31, n. 2, p. 100-104, 2004.

RAPELLO, G. V. G. et al. Pulmonary emphysema induced by methylphenidate: experimental study. Sao Paulo Med J, v. 133, n. 2, p. 131-134, 2015.

ROCHA, F. L.; MALLOY-DINIZ, L. F.; HARA, C. Emprego de metilfenidato para o tratamento de déficit cognitivo em paciente com seqüela de traumatismo cranioencefálico. J bras psiquiatr, v. 55, n. 1, p. 78-81, 2006.

SALVIANO, L. H. M. S. Estudo de segurança da Ritalina® (cloridrato de metilfenidato) em animais adultos: aspectos de neurotoxicidade $\mathrm{e}$ nefrotoxicidade. Tese de doutorado - Universidade de Federal do Ceará, Fortaleza, p. 149. 2015.

Soumis : Mai 2021.

Approuvé : Mai 2021. 